\title{
Geometric Properties of Crumpled Wires and the Condensed Non-Solid Packing State of Very Long Molecular Chains
}

\author{
Marcelo A. F. Gomes, ${ }^{*, a}$ Valdemiro P. Brito ${ }^{b}$ and Maycon S. Araújo ${ }^{b}$ \\ ${ }^{a}$ Departamento de Física, Universidade Federal de Pernambuco, \\ 50670-901, Recife - PE, Brazil \\ ${ }^{b}$ Departamento de Física, Universidade Federal do Piauí, \\ 64049-550, Teresina - PI, Brazil
}

\begin{abstract}
Aspectos geométricos associados com o empacotamento hierárquico e heterogêneo de arames amassados são revistos. $\mathrm{O}$ fenômeno recentemente descoberto de condensação de energia elástica de curvatura nessas estruturas é discutido e novos resultados são apresentados, com ênfase em leis de escala robustas. Examina-se a possível relevância destas leis nas propriedades conformacionais de cadeias moleculares longas e densamente empacotadas em estado não-sólido como observado, por exemplo, para o DNA nos cromossomas ou nas cápsulas virais. Em particular, argumentos de campo médio são usados para estimar a dependência entre o número de laços e o comprimento dessas cadeias.
\end{abstract}

Geometric aspects associated with the hierarchical and heterogeneous packing of crumpled wires are reviewed. The recently discovered phenomenon of condensation of elastic energy of curvature in these structures is discussed, and new results are presented with emphasis on robust scaling laws. It is examined the possible relevance of these laws in the conformational properties of long molecular chains densely packaged in a non-solid state, as e.g. in the packing of DNA strands in chromosomes, or in virus capsids. In particular, mean field arguments are used to estimate the dependence of the number of loops in the dense non-solid packed three-dimensional configurations of a very long polymer strand as a function of the number of monomers or the chain length.

Keywords: crumpled wire, scale invariance, DNA packing

\section{Introduction}

The last two decades have witnessed a growing interest in crumpling from the point of view of theory and experiments in physics, as well as from the point of view of applications in related sciences and technology. ${ }^{1-8}$

Recent experiments using very large ensembles of a single piece of wire irreversibly or semi-irreversibly crumpled by diverse means have revealed the existence of a new state of packing of matter which is characterized by the formation of rigid, hierarchical and heterogeneous structures of loops, obeying robust scaling laws, and presenting anomalous physical properties, with slowly decreasing average density as the size grows. ${ }^{9}$ In all these packing experiments, the wire is injected into a rigid cavity simultaneously from each one of two opposite channels, as can be observed from Figure 1.

*e-mail: mafg@ufpe.br
The cavities are carved in compact blocks of Plexiglas, glass or gypsum. All the internal measurements of the cavities have a typical uncertainty of $0.1 \mathrm{~mm}$. The injection of wire is manual at a typical constant rate of $v \approx 1 \mathrm{~cm} \mathrm{~s}^{-1}$. However, the experiments have shown that the statistical scaling functions describing the basic geometric aspects of these packing dynamics are essentially independent of $v$, as well as of the angle of injection defined by the two channels, of the materials, of the fluctuations in the injection from each one of the channels, and of the state of lubrication of the cavities. ${ }^{9}$ Some examples of such structures of crumpled wires are clearly observed in Figure 1-3: in the first Figures we show the pattern obtained from the forced injection of a copper wire with $1 \mathrm{~mm}$ of diameter into a quasi-twodimensional cavity of Plexiglas of $100 \mathrm{~mm}$ of radius and $1.1 \mathrm{~mm}$ of height. Figure 2 presents a somewhat different cascade of loops obtained with the same method as in Figure 1, but for a more plastic wire of the alloy $\mathrm{Sn}_{0.60} \mathrm{~Pb}_{0.40}$ 


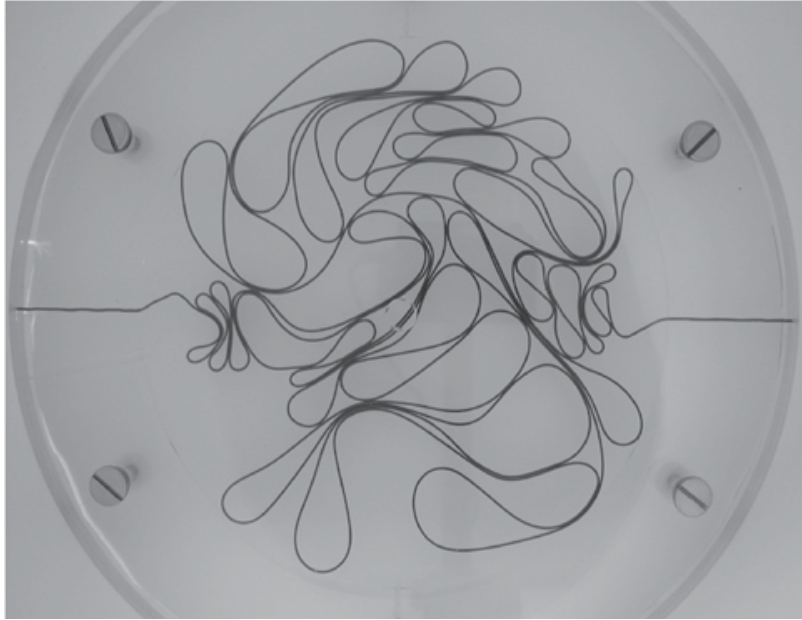

Figure 1. Example of heterogeneous hierarchical structures of loops obtained from the forced injection of a wire of copper with $1 \mathrm{~mm}$ diameter in a quasi-two-dimensional cavity of $100 \mathrm{~mm}$ of radius, and $1.1 \mathrm{~mm}$ of height.

(1.5 $\mathrm{mm}$ of diameter) in a quasi-two-dimensional cavity of $75 \mathrm{~mm}$ of radius and $1.6 \mathrm{~mm}$ of height. Figure 3 exhibits the entangled state obtained with the same type of wire as in Figure 2, but in a three-dimensional cavity of 75 $\mathrm{mm}$ of radius (the upper hemisphere of the cavity was removed to expose the structure). Interestingly, in all these examples the injection of wire is arrested when a critical volume fraction is reached. ${ }^{9}$ This critical value is largely independent of the forces and materials employed in the process, and it is less than the typical volume fraction of the usual condensed matter occurring in the granular, liquid or solid states.

The mass-size relation is one of the most basic geometric measures that we can assign to complex physical structures. In particular, for folded structures with the topology of the line, it measures the mass (or e.g. the number of monomer

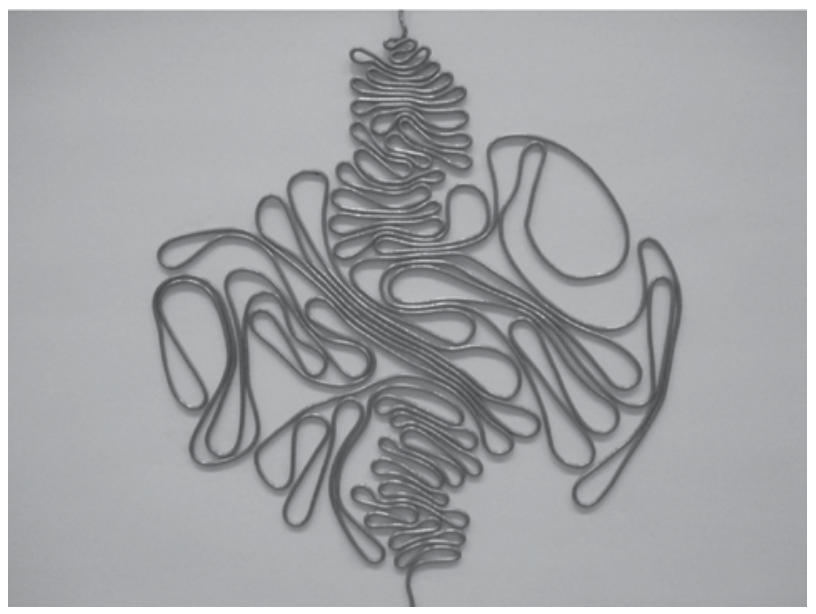

Figure 2. The same as in Figure 1, but for a wire of the alloy $\mathrm{Sn}_{060} \mathrm{~Pb}_{040}$ with $1.5 \mathrm{~mm}$ of diameter in a quasi-two-dimensional cavity of $75 \mathrm{~mm}$ of radius, and $1.6 \mathrm{~mm}$ of height.

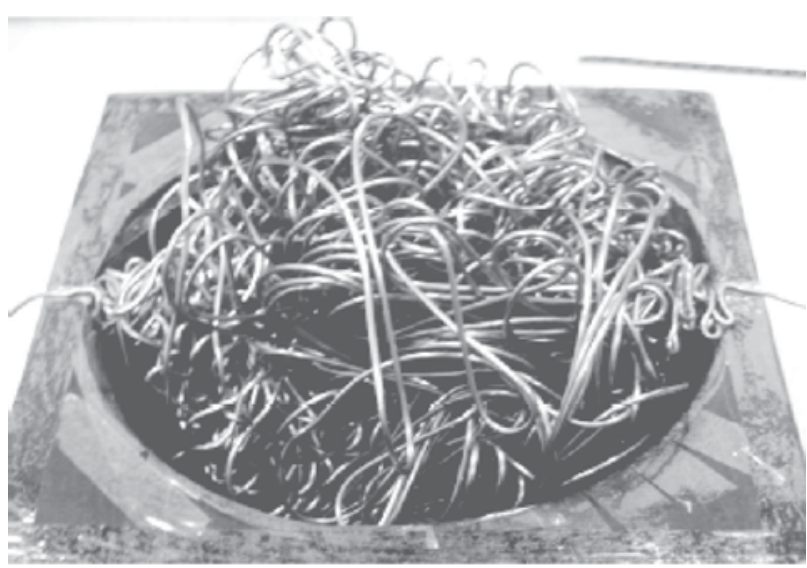

Figure 3. Example of a three-dimensional packing of wire obtained with the same method as in Figures 1 and 2, for the alloy wire of Figure 2, but in a spherical cavity of $75 \mathrm{~mm}$ of radius (the upper hemisphere of the cavity was removed to expose the structure).

units in a polymer), $M\left(R_{0}\right)$, within a sphere of radius $R_{0}$ with center at the center of mass of the system. If scale invariance is satisfied, it can be said that,

$M\left(R_{0}\right) \sim R_{0}{ }^{D}$

with $D$ being, in principle, a fractionary exponent interpreted as the fractal mass dimension of the system. ${ }^{10}$ Equation 1 satisfies the dilation (scaling) symmetry: $M\left(\lambda R_{0}\right)=\lambda^{D} M\left(R_{0}\right)$ for all positive value of the scale factor $\lambda$. Scale invariance with an anomalous non-integer exponent $D$ is frequently found in complex-structured systems, as e.g. in self-avoiding random walks (SARW) and polymers in general, as well as in percolation clusters, and crumpled cream layers. ${ }^{11-13}$ Thus, if $D$ is smaller than the dimensionality of the space, $d$, the average density of mass $\rho \sim M / R^{d} \sim R^{D-d}$ decreases anomalously as a power law in $R$. In Figure 4 we exhibit a typical masssize plot for an ensemble of 10 replicas of the crumpled structures exemplified in Figure 2, for $2 D$ cavities of radii $R_{0}(\mathrm{~cm})=1.00 ; 1.50 ; 2.50 ; 5.00 ; 7.50$; and 11.0 . The mass in this case is measured in units of length: $M \sim L$, where $L$ is the total length of wire injected in the cavity. From this $\log -\log$ plot we obtain $L \sim R_{0}{ }^{D}$, with $D=1.8 \pm 0.1$. Figure 5 shows the corresponding mass-size plot for crumpled structures of wire in three-dimensional cavities of radii $R_{0}(\mathrm{~cm})=0.90 ; 1.25 ; 1.7 ; 2.0 ; 2.50$; 5.00; and 7.50. In this case, we get $M \sim L \sim R_{0}{ }^{D}$, with $D=2.7 \pm 0.1$. In the three-dimensional physical space, crumpled balls of paper or metal foils, and crumpled wires squeezed manually and irreversibly into approximately globular compact units (i.e. using the hands as a cavity) obey scaling (1) with the mass fractal dimension assuming, respectively, the values $D=2.50 \pm 0.20$, and $D=2.7 \pm 0.2$, 


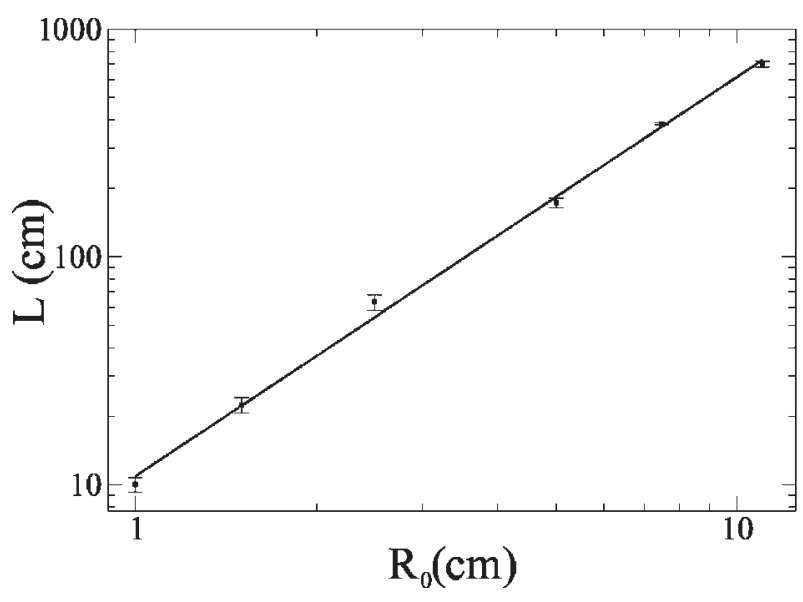

Figure 4. Mass-size plot for an ensemble of 10 replicas of the crumpled structures exemplified in Figure 2. The mass in this case is measured in units of length: $M \sim L$, where $L$ is the total length of wire injected in the cavity (Two-dimensional cavities of radii $R_{0}(\mathrm{~cm})=1.00 ; 1.50 ; 2.50$; 5.00; 7.50 and 11.0.). The continuous line represents the best fit $L \sim R_{0}{ }^{D}$, with $D=1.8 \pm 0.1$. In this case the average density decays slowly as $R_{0}^{-(0.2 \pm 0.1)}$ (see text).

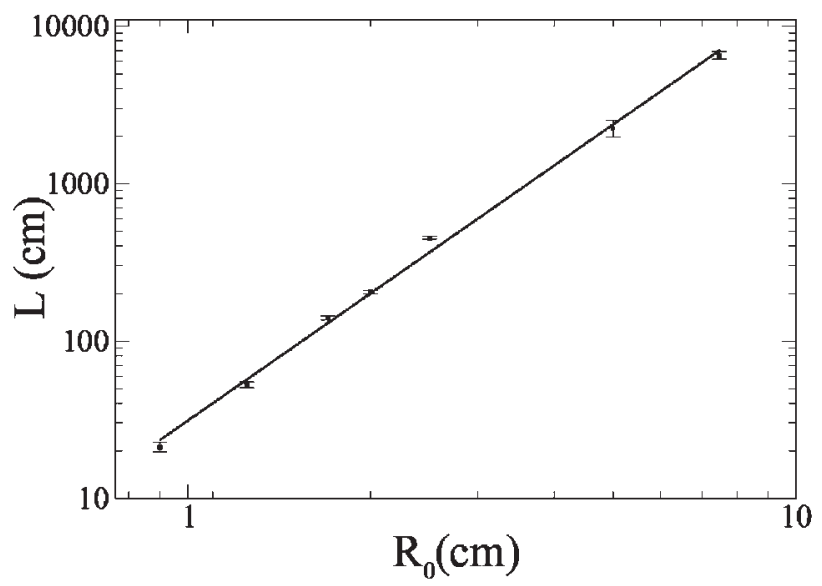

Figure 5. Same as in Figure 4, for three-dimensional cavities of radii $R_{0}(\mathrm{~cm})=0.90 ; 1.25 ; 1.7 ; 2.0 ; 2.50 ; 5.00$ and 7.50 . In this case, the best fit (continuous line) is $M \sim L \sim R_{0}{ }^{D}$, with $D=2.7 \pm 0.1$, and the average density decays slowly as $R_{0}^{-(0.3 \pm 0.1)}$ (see text).

regardless the details of the process and of the materials, and along several orders of magnitude in mass. ${ }^{14,15}$

Experiments show that the crumpled structures of wire present a pronounced invariance of the mass-size dimension not only for globular shapes, but also for highly compressed axial (oblate) deformations at least up to $65 \%$, i.e. if the size of the system along the compression axis is typically reduced to $1 / 3$ of its original magnitude. ${ }^{15}$

\section{Discussion}

\section{Estimating the mass-size exponent for crumpled wires}

The mass-size exponent for a $d$-dimensional ball of crumpled wire can be easily estimated from the following mean field argument. Let us assign a total energy $U$ to a ball of crumpled wire of length $L$ and radius $R$, with mass $M \sim L$. This total energy is divided in the most coarse-grained way possible in two contributions: (i) A term coming from a simple parabolic confining energy, $U_{c}=k R^{2} / 2$, where $k>0$ is an effective constant. This term corresponds to an inward linear force $F_{c}=-\partial U_{c} / \partial R=-k R$. (ii) The second term is a self-avoidance energy $U_{s a}$, which is assumed to be a twobody interaction, and consequently is proportional to the square of the average density of mass, $\rho \sim\left(M / R^{d}\right)$, where the $d$-dimensional volume scales as $V \sim R^{d}$. This self-avoidance energy term thus reads after integration in the volume: $U_{s a} \sim$ $\rho^{2} \times V \sim M^{2} \times R^{-d}$. This energy corresponds in turn to an outward nonlinear force $F_{s a}=-\partial U_{s a} / \partial R \sim M^{2} R^{-(d+1)}$. The confining term, which can be associated with entropic, packaging and segregation forces, tends to privilege a packed configuration of the structure, while the self-avoidance term, which has its microscopic origin in Pauli's exclusion principle, tends to privilege extended configurations of the system. In our model, $F_{s a}$ simulates the important steric repulsion forces, which is one of the dominant forces in molecular physics at the microscopic level. By minimizing the total energy $U=U_{c}+U_{s a}$ with respect to the globular radius $R$, we obtain immediately the mass-size $\left(R_{0}\right)$ dependence at the equilibrium as the power law $M \sim R_{0}^{D}$, with $D=(d+2) / 2$, independently of the values of the coupling constants associated with $U_{c}$ and $U_{s a}$. Thus, $D=2$ (2.5) for $d=2$ (3), in reasonable agreement with the data reported in the Introduction for crumpled wires in two- and three-dimensional space, within typical statistical fluctuations of $10 \%$. Contrarily to the model prediction for $d=3$, if this crude mean field model is applied to the twodimensional packing of wire, no diminution of the average density with $R_{0}$ is expected: $\rho \sim R^{0}$. As it is known, mean field results are, in general, expected to ameliorate with the increase of $d$, and a breakdown of mean field tends to occur at low dimensionality. However, the result $D(d=2)=2$, obtained with the simple model discussed here is almost spectacularly good as compared with the experimental value $D=1.8 \pm 0.1$. Furthermore, the minimum configurational energy of the structure is given by $U_{\min } \sim M^{4 /(d+2)} \sim L^{4 /(d+2)}$, a result which can be tested experimentally. If it is conjectured that the last result is valid at the level of a chromosome, with the DNA strand developing the role of wire, it could be tested in experiments of manipulation of a single DNA packed structure as the work done to stretch the long molecular chain from that condensed state. ${ }^{16}$

\section{Condensation of elastic energy in crumpled wires}

It was shown recently that the elastic energy in twodimensional packing of crumpled wires is condensed in 
a low-dimensional support. ${ }^{17}$ That is, in these systems, the elastic energy of curvature is concentrated on a set whose experimental value of the dimension is close to the unit $(D=1.0 \pm 0.1)$, whereas the mass, as explained in the previous section, is distributed on a set with larger dimension, $D=1.8 \pm 0.1$. The elastic energy due to the stretching in this problem is negligible as compared with the elastic energy of curvature. Thus, the stored elastic energy of curvature, $E$, for a crumpled wire packaged in a cavity of radius $R_{0}$ scales as $E \sim R_{0}^{\beta}$, with $\beta=1$, in the limit of maximum packing density. It has been conjectured that this numerical value of $\beta$ remains valid as well in threedimensional packing of crumpled wires. ${ }^{17}$ The reason of this decoupling between mass and energy of curvature lies in two facts. First, energy is effectively stored only in the bulge-extremity of the loops (see e.g. Figure 1): the elastic energy of curvature is inversely proportional to the square of the radius of curvature, and this radius is smaller just in the bulge of each loop. Secondly, there is a hierarchy of loops distributed heterogeneously within the cavity, and smaller loops, presenting bulges with small radius of curvature, tend to give a larger contribution to the stored elastic energy of curvature. As a consequence, the elastic energy is heterogeneously distributed in the space. Contrarily, in a more familiar system as a usual spring, the elastic energy is homogeneously distributed in the space, and it accompanies the distribution of mass. It is interesting to observe that if we try to fill regularly and completely a circular cavity with an elastic wire in order to obtain a maximum volume fraction close to the unit (in a configuration where segments of the wire are parallel, and touch their neighbors), the regions of the wire displaying the smaller radii of curvature stay in the perimeter of the cavity (on the circumference), that is, on a one-dimension subset of the plane. The difference between this last particular situation and that considered in the first paragraph (Figures 1 and 2) is that in those figures the structures are heterogeneous, hierarchical, and have less volume fraction, whereas the other is homogeneous, regular and non-hierarchical, and has volume fraction very close to the unit. In the next section, using a mean field approximation, it is made a theoretical estimate of the implications of the condensation of elastic energy of curvature on the packing properties of crumpled wires, and on the packing of DNA-like molecular chains.

\section{Crumpled wires and packing of DNA-like molecular chains}

As it is well known, details are important in biology and ultimately the structure of biological macromolecules can be determined to atomic resolution and can be related to the way that the molecules function. On the other hand, statistical aspects and mathematical modeling of DNA packing are presently topics of great interest for molecular biology as well. ${ }^{18}$ Very recently it has been introduced the use of paper models of crumpled surfaces as simple analogical tools for the biologist to develop insight into the statistics of the geometric intricacies of the packing of complex biological structures as membranes and proteins. ${ }^{7}$

Although historically there has been a strong focus on the conformation of "open" DNA backbones, many aspects of DNA highly packed structures are presently been studied. ${ }^{19}$ Here, crumpled wires with globular or oblate shape, obtained with a piece of wire irreversibly folded by manual means, are used to model the packing of DNA-like molecular chains in confined geometries, as those observed for DNA chains in chromosomes or virus capsids.

Let us consider a very long linear molecule of unfolded length $L$ as a non-ramified polymer-like chain with mass $M \sim L \sim R_{0}^{D}$, i.e. obeying the mass-size scaling (1), with $R_{0}$ being the length scale in the three-dimensional packed configuration. As an illustration, from data reported in the literature we find that the genome length $L$ within a real bacteriophage viral capsid of size $R_{0}$ scales as $L \sim R_{0}{ }^{D}$, with $D=1.9$, with a correlation of $90 \%$ (similar results are valid for other types of viruses). ${ }^{20}$ This mass fractal dimension has within the fluctuation bars the same value found for crumpled structures obtained in forced packing of a long metallic wire injected into a two-dimensional cavity. To maximize the packing density within small volumes, a very long molecule must present conformations with a certain number $N$ of loops or folds associated with small radii of curvature. A loop, in the context of the present work, is any segment with a round or oval shape, formed by a curve that is closed or approximately closed and does not intersect itself. Thus, the loops contain the regions associated with the smallest curvature radii in the packed molecular structure. Using the scaling hypothesis

$N \sim L^{\alpha}$

and considering that there is an energy cost $\varepsilon \sim(1 / r)^{2}$ to create a single loop with radius of curvature $r$, we can estimate the total elastic energy of curvature stored in the packing as $E=N \varepsilon \sim N^{3} / L^{2}$, where in the last scaling relation we adopted for the curvature radius the mean-field value $r \sim L / N$. Using the previous relations we express $E$ as a function of the chain length $L$, or $R_{0}$ :

$E \sim L^{3 \alpha-2} \sim R_{0}^{\beta}, \beta=D(3 \alpha-2), \beta \leq D$

If (3) is valid, we get for the exponent $\alpha$ introduced in (2) the value $\alpha=(\beta+2 D) / 3 D$, and the expected number 
of loops, $N$, as a function of $L$ or $R$ could be obtained and verified experimentally:

$N \sim L^{(\beta+2 D) / 3 D} \sim R_{0}^{(\beta+2 D) / 3}$

This is in fact verified for crumpled wires, as shown in Figure 6 which gives the number of loops $N$ as a function of the radius of the cavity $R_{0}$ for two-dimensional packing of wires of the type illustrated in Figures 1 and 2. In this particular case, the best fit (continuous line) represents the scaling $N \sim R_{0}^{1.30}$, a result in agreement with (4), and with the numerical values $\beta=1$, and $D=1.8$, within error bars of $10 \%$ or less. In principle, $0<\beta \leq D \leq 3$, and consequently $2 / 3<\alpha<1$. A compact system $(D=3)$, with elastic energy of curvature homogeneously distributed throughout the volume $\left(\beta=3\right.$ ), has $N \sim L^{1} \sim R_{0}^{3}$ (it can not be forgotten that the topology in the present article is always one-dimensional). In the opposite limit of a very tenuous system, with both $D$ and $\beta$ small, a smaller value for $\alpha$ is obtained. If the packing of mass is optimized, and $D \rightarrow 3$, but the elastic energy is condensed, with $\beta \approx 1$, as discussed in the previous section and observed for crumpled wires, we get $N \sim L^{7 / 9} \approx L^{0.77}$. This last result, which represents a deviation from the homogeneous limit $N \sim L$, could be experimentally tested with the present techniques..$^{16,20}$ Progressive condensation of elastic energy of curvature on sets of low dimensionality means that the total number of loops in the structure grows slowly as a nonlinear power law of the chain length $L$.

Using the value $D=1.9$ reported in the beginning of the previous paragraph in equation (4), together with $\beta=1$, we get $\alpha=0.84$; i.e. the number of loops in the model bacteriophage viral capsids discussed here will scale as $N \sim L^{0.84} \sim R_{0}{ }^{1.6}$. Equally, the average loop size $\lambda=L / N \sim L^{1-\alpha} \approx L^{0.16}$ will increase slowly as a power law of the genome length, whereas for homogeneous systems ( $\beta=D=3$ ) this quantity is independent of the genome length (or varies as $\log L$ ).

\section{Conclusions}

It is conjectured that experiments with crumpled wires can provide new insights into the issue of DNA packing. In both classes of systems two aspects are of fundamental importance: firstly, the self-avoiding interaction, simulating steric constraints of nonlocal origin, is always present, and secondly, the quasi-isometry and the one-dimensional topology is maintained fixed. These few ingredients seem to restrict considerably the allowed geometric configurations of a stringlike structure in equilibrium as well as in its dynamic behavior, irrespective the peculiarities associated

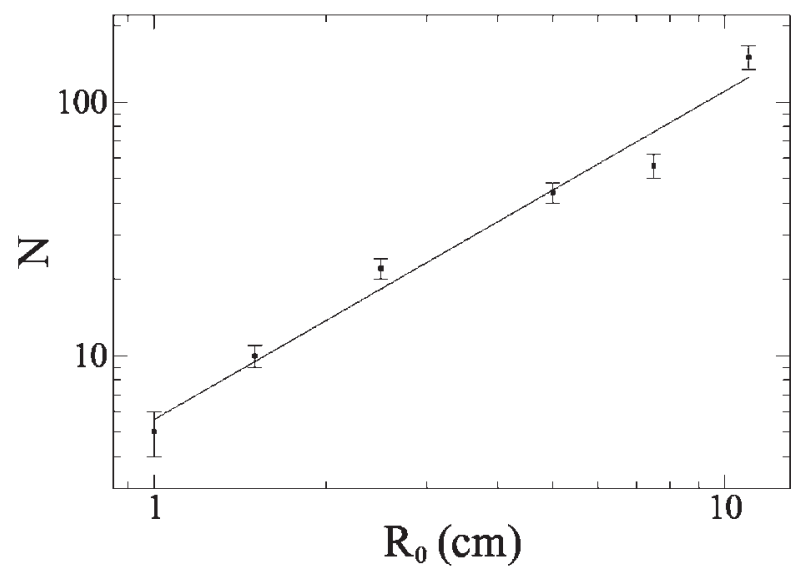

Figure 6. Number of loops as a function of the radius of the cavity for two-dimensional packing of wires of the type illustrated in Figures 1 and 2 . The best fit (continuous line) represents the scaling $N \sim R_{0}^{1.30}$, a result in agreement with equation 4 , and with the numerical values $\beta=1$, and $D=1.8$, within error bars of $10 \%$ or less.

with boundary conditions and material constitution. Although it can be verified experimentally that many DNA condensates are in toroidal, spiral, helical, and other regular conformations, we believe that other geometric types of structures of DNA condensates are possible, as those discussed in the present work in connection with the anomalous hierarchical structures of crumpled wires.

\section{Acknowledgments}

Financial support from CNPq and PRONEX (Brazilian Agencies) is acknowledged. We are grateful to Dr. C. C. Donato for Figure 1.

\section{References}

1. Gomes, M. A. F.; Am. J. Phys. 1987, 55, 649.

2. Kantor, Y.; Kardar, M.; Nelson, D. R.; Phys. Rev. Lett. 1986, $57,791$.

3. Wen, X.; Garland, C. W.; Hwa, T.; Kardar, M.; Kokufuta, E.; Li, Y.; Orkisz, M.; Tanaka, T.; Nature 1992, 355, 426.

4. Lobkovsky, A.; Gentges, S.; Li, H.; Morse, D.; Witten, T. A.; Science 1995, 270, 1482.

5. Chaieb, S.; Natrajan, V. K.; Abd El-rahman, A.; Phys. Rev. Lett. 2006, 96, 078101.

6. Vliegenthart, G. A.; Gompper, G.; Nat. Mater. 2006, 5, 216.

7. Cassia-Moura, R.; Gomes, M. A. F.; J. Theor. Biol. 2006, 238, 331.

8. Witten, T. A.; Rev. Mod. Phys. 2007, 79, 643.

9. Donato, C. C.; Gomes, M. A. F.; de Souza, R. E.; Phys. Rev. E 2003, 67, 026110.

10. Feder, J.; Fractals, Plenum: New York, 1988.

11. Havlin, S.; Ben-Avraham, D.; Phys. Rev. A 1982, 26, 1728. 
12. Stauffer, D.; Aharony, A.; Introduction to Percolation Theory, Taylor and Francis: London, 1994.

13. Gomes, M. A. F.; Donato, C. C.; Campello, S. L.; de Souza, R. E.; Cassia-Moura, R; J. Phys. D: Appl. Phys. 2007, 40, 3665.

14. Gomes, M. A. F.; J. Phys. A: Math. Gen. 1987, 20, L283.

15. Gomes, M. A. F.; Lima, F. F.; Oliveira, V. M.; Philos. Mag. Lett. 1991, 64, 361 .

16. Strick, T.; Allemand, J.-F.; Croquette, V.; Bensimon, D.; Phys. Today 2001, 54, 46 .
17. Donato, C. C.; Gomes, M. A. F.; Phys. Rev. E 2007, 75 , 066113.

18. Katzav, E.; Adda-Bedia, M.; Boudaoud, A.; Proc. Natl. Acad. Sci. USA 2006, 103, 18900.

19. Johnson, J. E.; Chiu, W; Curr. Opin. Struct. Biol. 2007, 17, 237.

20. Purohit, P. K.; Inamdar, M. M.; Grayson, P. D.; Squires, T. M.; Kondev, J.; Phillips, R.; Biophys. J. 2005, 88, 851.

Received: August 21, 2007 Web Release Date: November 30, 2007 